\title{
Anabases
}

ANABASES Traditions et réceptions de l'Antiquité

18 | 2013

Varia

\section{Paysages et topoi dans le péplum grec contemporain : de l'esthétique de la ruine à sa résurrection virtuelle}

Laury-Nuria André et Sophie Lécole Solnychkine

\section{(2) OpenEdition \\ Journals}

Édition électronique

URL : http://journals.openedition.org/anabases/4425

DOI : $10.4000 /$ anabases.4425

ISSN : 2256-9421

Éditeur

E.R.A.S.M.E.

\section{Édition imprimée}

Date de publication : 1 octobre 2013

Pagination : 109-127

ISSN : 1774-4296

\section{Référence électronique}

Laury-Nuria André et Sophie Lécole Solnychkine, « Paysages et topoi dans le péplum grec contemporain : de l'esthétique de la ruine à sa résurrection virtuelle ", Anabases [En ligne], 18 | 2013, mis en ligne le 01 novembre 2016, consulté le 21 octobre 2019. URL : http://journals.openedition.org/ anabases/4425; DOI : 10.4000/anabases.4425 


\title{
Paysages et topoi dans le péplum grec contemporain : de l'esthétique de la ruine à sa résurrection virtuelle
}

\author{
Laury-Nuria André, Sophie Lécole Solnychkine
}

"Par définition, la ruine est un fragment. Les pierres du Forum, les colonnes du temple, les tours de la cathédrale sont les vestiges d'un monument disparu, d'une histoire qui n'est plus. La ruine évoque donc l'absence. Et pourtant, dans le même instant pourrait-on dire, par sa présence, la ruine impose son propre univers, avec ses couleurs, son ambiance, ses fantômes, et finit par se détacher de son passé, comme une feuille du calendrier. Aussi, la ruine est plus qu'un fragment ; son autonomie, qu'écrivains et artistes ont fini par instaurer, lui donnent un statut à part entière ${ }^{1}$. "

D ANS LES CHOIX QUI PRÉSIDENT à la mise en scène de l'Antique ${ }^{2}$ dans le cinéma contemporain, une attention particulière est portée à la représentation de l'espace diégétique. Ce dernier participe, au même titre que les costumes (dont vient d'ailleurs le terme

1 M. Makarius, Ruines, Représentations dans l'art de la Renaissance à nos jours, Paris, Flammarion, 2011, p. 167.

2 Comme nous allons le voir, notre corpus se constitue de films dont certains relèvent à proprement parler du péplum, tandis que d'autres s'inscrivent davantage dans la fantasy antique ou dans la biographie historique. La question de la délimitation générique n'étant pas ici notre propos, nous parlerons plus largement de "fictions antiquisantes ", afin de questionner la mise en scène de l'Antique au cinéma. 
générique péplum ${ }^{3}$ ) de la possibilité, pour le spectateur, d'identifier géographiquement et temporellement le cadre de l'action. Paysages naturels et urbains sont alors bien plus que de simples décors. Destinés à camper une identité antique (la " couleur locale " à laquelle il faudrait imaginer un pendant temporel), ils font l'objet d'un investissement cinématographique dans lequel le spécialiste de l'Antiquité et le critique contemporain peuvent lire la trace d'une évolution de la réception de la matière antique.

Si l'architecture des palais fait, le plus souvent, l'objet d'un traitement vraisemblable, engageant un travail conséquent autour des décors de studio, on constate que dans la plupart des péplums jusqu'aux années 2000, le paysage est quant à lui parsemé de ruines. Il est en effet fréquent de rencontrer dans les films, au détour d'un chemin, quelques ruines éparses dans le paysage, comme si le signe visuel de l'Antiquité ne pouvait être que l'inscription, sur la pellicule, du temps qui nous sépare d'elle. La présence - dans le même temps que ces éléments ruinés qui ne peuvent logiquement résulter de l'usure de siècles ne s'étant pas encore écoulés - d'architectures correspondant au temps et au lieu de la diégèse, fait de ce paysage antique un paradoxe temporel. Malgré ce caractère paradoxal, la ruine - héritage esthétique ou stéréotype - figure le signe indépassable, inscrit dans l'espace et le paysage cinématographiques, de l'Antiquité. Toutefois, l'observation de la représentation des ruines dans les péplums historiques, et, plus largement, dans les films antiquisants, montre que si l'utilisation de la ruine afin de «signifier » l'antique est une constante jusqu'aux années 2000, la décennie 2000-2010 marque quant à elle un tournant de taille.

Comment comprendre en effet le passage notable, dans les films antiquisants de cette décennie, héritiers du péplum classique ${ }^{4}$, d'une utilisation de la ruine " ruinée 5 " comme signe de l'Antique à une reconstitution virtuelle " totale " du paysage et des espaces antiques canoniques ? C'est qu'il faut voir ici la marque d'un intérêt des réalisateurs pour l'utilisation des nouvelles technologies de l'image, lesquelles permettent des reconstitutions virtuelles détaillables à l'infini ${ }^{6}$, comme c'est le cas

3 Ainsi que l'explique Claude Aziza dans "Le mot et la chose ", in Cl. Aziza (dir.), Le péplum : l'Antiquité au cinéma, CinémAction 89 (1998), p. 7-11.

4 Nous renvoyons le lecteur aux ouvrages de Claude Aziza, notamment au Guide de l'Antiquité imaginaire, Paris, Les Belles Lettres, 2008 ; et Péplum, un mauvais genre, Paris, Klincksieck, 2009.

5 Nous souhaitons ici distinguer la ruine, résultat " naturel " de l'usure du temps, des destructions, etc., de la ruine " artefactuelle ", soit celle que l'on construit dans une perspective décorative ou esthétique (art des jardins, cinéma, décor de théâtre, voire urbanisme si l'on pense aux travaux de James Wines pour la Site Agency, notamment à Ghost Parking Lot). Cette dernière, nous proposons de la nommer " ruine ruinée ».

6 De façon parallèle, on observe dans l'archéologie et les sciences de l'Antiquité un recours massif aux technologies de l'image afin de mettre en ouvre d'impressionnantes reconstitutions virtuelles. Ainsi on note que, grâce à ces techniques, l'archéologie s'attache de plus en plus à la reconstitution de l'état originel du matériel sur lequel elle travaille : recherche de la polychromie originelle de la statuaire, reconstitution 3D de temples ou 
par exemple pour la Bibliothèque d'Alexandrie dans Agora, ou encore pour l'Argos $\mathrm{du}$ Choc des Titans. Dès lors, c'est à une autre question, connexe, que l'analyse de ces villes antiques ressuscitées va nous mener. $\mathrm{Si}$, en effet, la technologie en son état permet la reconstitution $3 \mathrm{D}$ de villes entières ${ }^{7}$, elle s'offre aussi comme le moyen de manipuler des caméras virtuelles qui vont évoluer dans toutes les dimensions de ces espaces recréés, et ainsi permettre de filmer ces villes de manière inédite. Ainsi, c'est la question de la circulation du regard dans ces paysages virtuels qui devient prégnante, et que notre réflexion va envisager dans un dernier temps d'analyse.

Une telle interrogation sera menée dans le cadre d'un corpus cinématographique composé de péplums des années 2000 à 2010 : Troie (Wolfgang Petersen, 2004), Alexandre (Oliver Stone, 2004), Agora (Alejandro Amenábar, 2009), Le Choc des Titans (Louis Leterrier, 2010), Les Immortels (Tarsem Singh, 2011). Ce corpus présente l'avantage d'une unicité de fond mythologique : tous les films ici analysés réferent à la mythologie ou à l'histoire grecque. Étant l'une spécialiste du paysage grec épique antique et l'autre spécialiste du cinéma contemporain, nous avons souhaité conjoindre ici ces deux champs de recherche afin d'offrir une analyse transdisciplinaire de la question de la représentation du paysage antique au cinéma, à travers ce corpus de péplums à sujets grecs ${ }^{8}$.

\section{De la ruine ruinée à la Bibliothèque d'Alexandrie ressuscitée}

Le péplum traditionnel fait largement usage de ruines. Ainsi, par exemple, dans Jason et les Argonautes (Don Chaffey, 1963), la séquence où Hermès se présente à Jason pour le mener devant les dieux de l'Olympe se déroule dans un décor de fausses ruines : seules quelques colonnes se dressent encore vers le ciel, tandis que des vestiges de murs s'effondrent. Ce type de ruines, que l'on rencontre fréquemment dans le péplum traditionnel ${ }^{9}$, est aisément identifiable dans la mesure où il fait, curieusement, figure de

de palais, reconstitution de visages à partir d'une reconstruction tissulaire virtuelle de corps momifiés, etc.

$7 \quad$ Notons tout de même, dans la plupart des films de notre corpus, que l'utilisation des techniques de la 3D n'est pas exclusive. Les films associent en effet étroitement 3D numérique et $3 \mathrm{D}$ réelle dans le travail des décors.

8 Et ce, bien que de nombreux péplums sortis au même moment traitent de la culture romaine comme le célèbre Gladiator (Ridley Scott, 2000), ou Ben-Hur (Steve Shill, 2010), Centurion (Neil Marshall, 2010), ou encore L'Aigle de la Neuvième Légion (Kevin McDonald, 2011).

9 À notre connaissance, il n'existe pas de travaux de recherche portant précisément sur la question de la ruine dans les péplums ou les films antiquisants. Toutefois, il existe quelques études traitant des décors des films, ou de quelques éléments urbains précis (le forum, etc.) ; nous renvoyons le lecteur à trois articles de M. ÉLOY : "Archéologie et décors de cinéma : le Forum romain dans Cléopâtre ", in L'archéologie et son image, Actes des 
ruines " neuves ": les blocs de pierre taillée s'effondrent, mais les arêtes en sont aussi aiguës qu'au jour de la taille, des fûts de colonnes jonchent le sol, mais leurs cannelures sont intactes. Ces ruines artefactuelles nous semblent d'autant plus « neuves " que nous pouvons les comparer à de "vraies " ruines, lorsque, plus tard dans le film, la scène du repas de Phinée perturbé par les Érinyes, nous conduit sur le site archéologique du temple d'Héra I à Paestum. Ici, les cannelures sont érodées par le temps, et des mousses recouvrent partiellement la blancheur immaculée des pierres. Plus frappant encore, puisqu'il conjoint les deux types de ruines dans une même séquence, Le Choc des Titans (Desmond Davis, 1981) nous ramène au temple de Paestum précédemment cité (Héra II cette fois). En effet, la scène de l'entrée de Persée dans l'antre de Méduse ${ }^{10}$ est filmée dans les ruines véritables, tandis que le plan suivant nous confronte à de fausses ruines. Le décor de la caverne de Méduse jure d'autant plus avec les ruines archéologiques qu'il présente une esthétique orientalisante anachronique ${ }^{11}$ (et, vraisemblablement, déjà kitsch pour l'époque).

Qu'elles soient reconstruites ou archéologiques, les ruines relèvent dans ces deux cas de la même fonction diégétique : celle d'une temporalisation, dans des temps très anciens, de la trame narrative représentée.

C'est une utilisation similaire de la ruine que nous retrouvons dans les premiers péplums des années deux mille relevant de notre corpus. Ainsi, dans Troie, film de Wolfgang Petersen, sorti en 2004, une scène nous montre Achille, en plein entrânement avec Patrocle, à Phtie, région de Grèce constituant son royaume. C'est au milieu des ruines d'un temple placé sur les bords d'une falaise surplombant la mer que nous apparaît Achille. On voit rapidement qu'il s'agit de décors en carton-pâte et non de " vraies " ruines. Ceux-ci semblent pris en conflit entre une volonté de réalisme (" faire " antique) et une imprécision historique (puisque logiquement, ces ruines ne peuvent résulter de destructions ou du passage d'un temps n'ayant encore eu lieu). La ruine figure ici le signe de l'Antique, et relève d'un code esthétique qui permet, symboliquement, de transcrire une ambiance à l'Antique, de la rendre lisible au spectateur. Cette ruine " ruinée " saute d'autant plus aux yeux que la séquence précédant la scène

VIII Rencontres internationales d'archéologie et d'histoire d'Antibes, Centre de recherches archéologiques du CNRS \& Musée archéologique d'Antibes, Juan-les-Pins, éd. APDCA, 1988, p. 239-259 ; "Architecture et péplum. La cité antique, ou Le pouvoir de l'imaginaire ", in Architecture et cinéma, CinémAction 75 (1995) ; "La vision critique du péplum : le problème de la reconstitution ", in Revivre le passé grâce à l'archéologie / L'archéologie expérimentale, Les Dossiers d'archéologie 216 (1996), p. 52-57.

10 M. ÉLOY, «La mythologie grecque au cinéma », in Cl. AzIZA (dir.), Le péplum : l'Antiquité au cinéma, CinémAction 89 (1998), p. 29.

11 Nous pourrions par exemple rapprocher les colonnes à losanges figurées dans ce film de celles du monastère égyptien de Baouït reconstitué au Musée du Louvre (cf. N. BEL, C. Girolre, et alii (dir.), L'Orient romain et byzantin au Louvre, Paris, Louvre Éditions/ Actes Sud, 2012, p. 322-325). 
d'entraînement d'Achille et de Patrocle nous invite à découvrir le palais de Mycènes, lequel bénéficie d'un travail de décor studio qui le dote de l'épaisseur d'une réalité historique (il apparaît comme vraisemblable : en parfait état, non " ruiné »), bien que sa précision archéologique puisse être sujette à caution. C'est ainsi très agrandis, et curieusement situés au fond de la salle du trône du palais, que l'on retrouve les lions affrontés du triangle de décharge de la fameuse porte de Mycènes. Que penser de cette licence décorative quand on sait que la porte de Mycènes est l'un des monuments grecs les plus connus du grand public ? S'agit-il d'un jeu conscient de la part du réalisateur, qui pourrait ainsi - pourquoi pas ? - imaginer le remploi de ces œuvres lapidaires ${ }^{12}$ pour la célèbre porte, comme si les lions provenaient en fait des ruines d'un palais préexistant? Voilà un bel exemple de ce que nous pourrions appeler une fiction archéologique.

Cependant, ce ne sont pas les architectures palatiales, mais bien plutôt la reconstitution de la ville de Troie, en 3D numérique et 3D réelle, qui constitue le véritable morceau de bravoure du film. Nous trouvons en effet dans ce film une figuration de Troie à mi-chemin entre réalité historique et fiction archéologique. Cette reconstruction sollicite un imaginaire stéréotypé tout en s'appuyant sur une part de vérité plus ou moins importante : elle allie décor " réel » sur le plateau de tournage et décor numérique pour les plans larges saisissant l'ensemble du paysage urbain (scène de l'arrivée triomphale d'Hector, de Pâris et d'Hélène à Troie). S'il fait référence à l'épopée homérique, le film n'a pu en aucun cas s'appuyer sur l'Iliade afin de reconstruire la ville de Troie, celle-ci n'ayant pas chez Homère valeur territorialisante, mais plutôt générique : le poète en effet n'a pas décrit « le sac de la ville de Troie mais le sac de toute ville ${ }^{13} »$. Même si depuis, l'archéologie et le matériel iconographique antique permettent de renseigner sur ce qu'a pu être Troie, c'est un autre modèle qui a été

12 Au sujet du remploi des objets lapidaires, nous renvoyons le lecteur à l'article de L. Foulquier, La métamorphose des pierres. Les remplois, entre rebut et souvenir, in Histoire de l'art et anthropologie, Paris, INHA / Musée du quai Branly ("Les actes »), 2009, [En ligne], mis en ligne le 28 juillet 2009, Consulté le 19 septembre 2012. URL : [http:// actesbranly.revues.org/252].

13 C'est ce qu'a pointé G. Agosti lors de sa communication " Nonnus' Visual World " au colloque international Nonnus of Panopolis in Context, Poetry and Cultural Milieu in Late Antiquity, Rethymno, Crète, 13-15 mai 2011, à paraître. Cela signifie qu'il n'y a chez Homère nulle description détaillée de la ville de Troie, pas plus d'ailleurs que dans la littérature antique en son ensemble. On trouve cependant des indications urbanistiques chez Quintus de Smyrne, voir L.-N. ANDRÉ, Formes et fonctions du paysage dans l'épopée hellénistique et tardive, Thèse de doctorat sous la direction de Ch. Cusset, ENs de Lyon, 2012. Sur l'histoire de la ville de Troie, de son paysage et de sa représentation, voir A. TRACHSEL, La Troade : un paysage et son héritage littéraire. Les commentaires antiques sur la Troade, leur genèse et leur influence, Bâle, Schwabe, 2007. Pour les questions de pérennité de reconstruction et de résurrection de la ville de Troie, voir M. Fartzoff, M. Faudot, É. Geny, (éd.), Reconstruire Troie : permanence et renaissances d'une cité emblématique, Besançon, Presses universitaires de Franche-Comté, 2009. 
choisi dans ce film : le parti pris retenu est la convocation du modèle générique de la ville mésopotamienne, en l'occurrence Babylone. La porte principale de la ville, autour de laquelle se resserre l'action, semble inspirée de la célèbre porte d'Ishtar ${ }^{14}$. Dans les plans d'ensemble du paysage urbain, partiellement recomposé en 3D virtuelle, les toits en terrasse, les chapiteaux à décorations égyptiennes et les costumes orientalisants font alors " couleur locale ». Pour parvenir à ce résultat, le réalisateur et son équipe de décorateurs ont engagé un travail de collaboration avec des spécialistes du British Museum. Comme l'explique Wolfgang Petersen,

"J'étais plus intéressé par la reconstitution du monde antique de 1200 avant JésusChrist. À quoi ressemblait une ville, une bataille en ces temps-là ? [...] Il reste peu de traces de cette époque. On a trouvé pas mal de choses au British Museum. On a interprété le reste. Il est probable que beaucoup de choses que nous avons mises dans le film ne sont pas justes mais c'était un exercice intéressant ${ }^{15}$."

Dans ses propos, transparaît la difficulté de ménager la part du divertissement de large audience, et celle des nécessités relatives aux enjeux historiques et scientifiques. Toute la complexité du problème paysager que représentent les films de notre corpus se situe dans l'articulation entre ces deux pôles.

C'est pourtant une solution différente à ce problème qu'Oliver Stone choisit d'apporter à son Alexandre sorti la même année (2004), bien qu'on y retrouve la même opposition, topique, entre des ruines artefactuelles (celles dans lesquelles enseigne Aristote au jeune Alexandre et à ses camarades) et une architecture palatiale conjuguant $3 \mathrm{D}$ et $3 \mathrm{D}$ réelle. Cette dernière nous permet de découvrir les jardins en terrasse de Ptolémée Ir Sôter, la première Bibliothèque d'Alexandrie, située dans les quartiers royaux (Basileia), et une large vue sur le port de la ville laissant apparaître son célèbre phare. Si les ruines dans lesquelles professe Aristote relèvent de la même fonction que les ruines analysées jusqu’à présent, et constituent un simple marqueur temporel, un déictique de l'Antiquité, il est intéressant de noter qu'Oliver Stone singularise cette fonction par le recours à d'autres éléments visuels. Ces derniers ne sont pas à proprement parler paysagers, mais plutôt ornementaux (quoiqu'ils recoupent la question de la représentation du monde), et sont utilisés dans cette même logique de désignation temporelle. Il s'agit des mosaïques cartographiques, que l'on retrouve à plusieurs reprises et sous différentes formes dans le film. La première orne le mur de la Bibliothèque

14 Voir, entre autres, la reconstitution de Babylone au temps de Nabuchodonosor II faite à partir des données archéologiques rassemblées par R. Koldewey et W. Andrae entre 1899 et 1917, proposée par le tableau de Maurice Bardin, La Ville de Babylone, 1936, conservé au Chicago Oriental Institute, OIM P28752.

15 A. LorfÈVre, "De l'Iliade à Troie, un même souci de divertir ", Entretien avec W. Petersen, La libre Belgique, 11 mai 2004, URL = [http://www.lalibre.be/culture/cinema/ article/166128/de-l-iliade-a-troie-un-meme-souci-de-divertir.html], date de consultation le 27 septembre 2012. 
d'Alexandrie, et la seconde se situe sur le sol du temple ruiné dans lequel enseigne Aristote. Les deux cartes portent des inscriptions en anglais en lieu et place d'inscriptions en grec ancien, et utilisent pour ce faire une police de caractère hellénisante afin de conforter le spectateur dans l'idée qu'il est bien confronté à un objet archéologique grec. Toutefois, même si l'énormité peut prêter à sourire, on remarque que les décorateurs, pour réaliser ces deux mosaïques, ont fait l'effort d'adopter la technique locale en vigueur à l'époque (en l'occurrence, la technique de la mosaïque de galets caractéristique de Pella pour la mosaïque située où professe Aristote ${ }^{16}$, et celle de tesselle pour la mosaïque qui se trouve dans la Bibliothèque d'Alexandrie ${ }^{17}$ ). Dans cet effort de réalisme, il faut souligner que l'emploi de l'une, puis de l'autre de ces techniques, est chronologique. En outre, les deux représentations cartographiques présentent une figuration vraisemblable de l'état des connaissances de la géographie de l'oikoumène à ce moment-là de l'Histoire. Ici, le traitement de ces éléments décoratifs - hellénisation de la police de caractère ou vieillissement de l'image ${ }^{18}$-, même s'il relève d'une singularité, s'inscrit dans la même logique que la ruine, et suggère l'éloignement temporel et la couleur locale antique.

D'autres films, plus récents, relèvent d'une autre logique. Ceux-ci se chargent en effet, là où le traitement des décors des décennies précédentes s'inscrivait simplement dans une logique temporalisante, d'une valeur symbolique, investie dans la diégèse des films. Le Choc des Titans ${ }^{19}$ particularise le travail de la ruine en la mêlant de naturel : nous y découvrons un antre de Méduse figuré sous les traits d'une grotte architecturée. Métamorphosant le statut de la ruine dont elle relève tout de même (les éléments archi-

16 On peut rapprocher cette mosaïque de galets de celles qui sont conservées au Musée archéologique de Pella : Dionysos sur une panthère, La chasse au lion, La chasse au cerf.

17 Les décorateurs ont ici utilisé l'opus tesselatum, technique caractéristique de la mosaïque hellénistique et romaine. La mosaïque de La bataille d'Alexandre contre Darius, conservée au Musée de Naples, sert ici de modèle. Il faut noter que les décorateurs l'ont représentée intacte, proposant ainsi une forme de restauration imaginaire non dénuée d'intérêt : un autre exemple de ces «fictions archéologiques" dont nous parlions plus haut.

18 On pense également ici au film Gladiator de Ridley Scott, qui, s'il excède notre corpus puisqu'il n'investit pas l'Antiquité grecque mais romaine, utilise (dans la scène du triomphe de Commode) un procédé de "vieillissement » des images très curieux qui leur donne un côté "images d'archives ", comme si on opérait un rabattement entre la première moitié du Xx siècle, à laquelle renvoie ce type d'images, et l'Antiquité. Toutefois, il s'agit vraisemblablement ici également d'une référence cinématographique, qui rappelle les images d'Hitler à Nuremberg que l'on voit dans le film de L. Riefenstahl (Le triomphe de la volonté, 1934). De cette manière, par ce jeu intertextuel, Ridley Scott identifie le caractère tyrannique de Commode à la figure dictatoriale d'Hitler, et souligne dans le même temps l'emprunt, par l'Allemagne nazie, de mises en scène du pouvoir politique et de symboles romains.

19 Film de Louis Leterrier, sorti en 2010, remake du film éponyme de Desmond Davis sorti en 1981 . 
tecturaux qui y figurent sont en ruine, tandis que la grotte ${ }^{20}$ participe d'un imaginaire de la ruine terrestre), celle-ci prend une valeur symbolique par laquelle le paysage n'est plus un simple marqueur temporel, mais devient un véritable acteur de la diégèse. L'espace diégétique du Choc des Titans s'organise, à première vue, selon une opposition paysagère entre les paysages urbains, lieux de l'expression du tragique humain, et les espaces naturels, lieux du périple et de la geste épique du héros Persée. Ces derniers sont constitués par deux types de paysages : on trouve d'un côté les paysages du périple épique à proprement parler : la forêt, la mer de nuages, les montagnes et les prairies traversées. De l'autre, on rencontre des paysages littéralement extraordinaires, qui vont former le cadre des actions héroïques de Persée : le désert, théâtre de l'épreuve aux scorpions, le jardin du Styx, celui des Sorcières et enfin l'antre de Méduse, autant d'espaces qui relèvent de la catégorie du topos épique (dans l'acception moderne du terme) et qui en proposent des actualisations contemporaines. Il faut ici noter que les paysages des actions héroïques participent de la geste du héros, qu'ils contribuent, du fait de leur nature, à éprouver : exogènes à l'oikoumène, ce sont des paysages de l'extrême, entre désert et Enfers.

Toutefois, l'un de ces espaces de la geste du héros marque dans le film de Louis Leterrier une singularité qui met en dialogue les deux éléments paysagers que nous tenions pour opposés : ainsi, l'antre de Méduse, grotte architecturée, met en présence artefacts en ruines et éléments minéraux naturels. On reconnaît dans ces fragments architecturaux les vestiges d'un bâtiment des plus connus, puisqu'il s'agit du Parthénon ${ }^{21}$, emblème de la ville d'Athènes depuis l'Antiquité classique jusqu'à nos jours. Accolé à la paroi rocheuse, un fronton classique dont les colonnes s'effondrent signale l'entrée de la caverne de Méduse. Évoquant les flammes infernales, une lueur luit entre ces effondrements, qui attire l'œil vers l'intérieur. Happé par la lumière, le héros pénètre dans cet espace désolé : il y découvre un enchevêtrement où gisent fragments de façade, colonnes épurées de style dorique, statues d'Athéna et morceaux de fronton du Parthénon. Caractéristique étonnante de cette grotte, l'espace s'y nivelle en paliers. Ceux-ci sont rythmés par la démultiplication des mêmes éléments ruinés, qui cette fois se prêtent à une lecture symbolique. Ici, la ruine prend une valeur nouvelle, catoptrique : non plus signe d'une temporalité chronologique, linéaire (celle qui nous sépare de l'Antiquité), elle figure ici la claustration dans un temps et un espace circu-

20 Nous renvoyons ici le lecteur à G. SiEBERT, "Imaginaire et images de la grotte dans la Grèce archaïque et classique ", in G. SIEBERT (éd.), Nature et paysage dans la pensée et l'environnement des civilisations antiques, Paris, De Boccard, 1996, p. 47-57 ainsi qu'à R. Buxton, La Grèce de l'imaginaire, les contextes de la mythologie, Paris, La Découverte, 1996, p. 117-131.

21 Construit par les Athéniens au v s., le Parthénon n'entretient pas de rapport avec le mythe de Persée, lequel réfere aux temps archaïques immémoriaux, et dont la première occurrence littéraire se trouve chez Homère (Iliade IV, 160). 
laires (ceux du mythe et de ses retours), où Méduse erre, confrontée en permanence à la figure, figée dans la pierre, de celle qui l'a punie.

En ce sens, dans Le Choc des Titans, la ruine change de plan de référence, en n'étant plus instrumentalisée à des fins de marquage temporel. De fait, libérée de sa fonction déictique, elle peut embrasser une valeur symbolique. C'est non seulement parce qu'elle passe du marquage temporel à l'enjeu symbolique, mais encore parce qu'elle engage un autre champ de référence esthétique (la représentation d'un chaos), que la ruine figurant l'antre de Méduse dans Le choc des Titans de 2010 marque une rupture notoire avec les ruines précédemment étudiées. Si ces dernières s'inscrivent dans le paradigme classique de la ruine dix-huitiémiste (une ruine relevant d'une conception winckelmannienne de l'Antiquité, pourrions-nous dire...), la ruine de la caverne de Méduse s'inscrit quant à elle dans un champ de référence tout autre. Nous sommes ici du côté de la désolation, du chaos. Cette ruine est aussi celle de notre fin de $\mathrm{XX}^{\mathrm{e}}$ siècle : elle s'identifie à un imaginaire nourri des images de paysages issus de la catastrophe (guerres mondiales et/ou nucléaires, accidents industriels ou écologiques).

Il est intéressant de constater que ce changement de plan de référence esthétique de la ruine n'est pas un unicum: on en trouve trace, sous une autre forme, dans Les Immortels. Dans ce film de Tarsem Singh, sorti en 2011, l'on retrouve le héros Thésée, qui reçoit, dans les moments les plus critiques de sa pérégrination, l'aide des dieux. Poséidon plonge dans la mer afin de provoquer une vague gigantesque qui engloutit les assaillants de Thésée, mais qui, en contrepartie, recouvre le littoral et les héros d'une curieuse substance gluante, laquelle a tout de la marée noire. Il est extrêmement étrange de rencontrer un imaginaire écofictionnel ${ }^{22}$ très contemporain dans une fiction antiquisante. On peut ainsi questionner la manière dont le péplum se fait le reflet des préoccupations sociétales des époques qui le produisent ${ }^{23}$.

Dans le même ordre d'idées, on a reproché au réalisateur espagnol Alejandro Amenábar d'avoir, dans le film Agora, instrumentalisé la vie d'un personnage historique, Hypatie d'Alexandrie, pour en faire, en plein conflit entre les États-Unis et le Proche-Orient, le lieu d'une diatribe contre le fanatisme religieux. Sa manière de filmer les chrétiens de l'Alexandrie de la fin de l'Antiquité, ainsi que le choix des acteurs devant interpréter les figures de pouvoir de la religion chrétienne (Cyrille d'Alexandrie, évêque fanatique d'Alexandrie, interprété par Sami Samir, et Ammonius, prêtre

22 Nous renvoyons le lecteur à l'ouvrage de Ch. Chelebourg, Les écofictions, mythologies de la fin du monde, Bruxelles, Les Impressions Nouvelles, 2012.

23 Ainsi, une lecture, relativement répandue dans la presse des années 2004-2005, a cru déceler, dans le film Troie de W. Petersen, précédemment cité, l'incarnation de G. W. Bush dans la figure tyrannique d'Agamemnon, en comparant le " prétexte " du ravissement d'Hélène afin d'entrer en guerre avec Troie au «prétexte » d'armes de destruction massive localisées en Irak. 
organisant la milice des parabalani et les missions d'évangélisation, campé par Ashraf Barhom) ont froissé, voire choqué l'opinion publique ${ }^{24}$.

Toutefois, au-delà du reflet de ces préoccupations contemporaines, le film Agora témoigne d'une réelle recherche esthétique et d'une utilisation de la ruine tout à fait intéressante pour notre propos. Deux exemples tirés du film Agora sont particulièrement révélateurs des possibilités d'une utilisation métaphorique des ruines, lorsque celles-ci se dégagent de leur simple signification temporelle. Sur l'agora, en effet, lorsque Ammonius s'oppose à son interlocuteur païen, on aperçoit des ruines (en l'occurrence des arcades romaines). Ensuite, lorsque Synésios de Cyrène revient à Alexandrie pour tenter d'apaiser les querelles soulevées par Cyrille, évêque fanatique d'Alexandrie, on distingue à nouveau dans les rues que celui-ci traverse des ruines, cette fois égyptiennes. Dans ces deux séquences, les ruines ont à la fois une valeur symbolique, puisqu'elles désignent le palimpseste d'identités païennes sur lesquelles la culture alexandrine s'est structurée (identité égyptienne conservée par le régime ptolémaïque, identité hellénistique gardée par l'Empire romain) et une valeur narratologique de prolepse. À chaque fois qu'il y a confrontation entre un personnage incarnant la culture païenne et un personnage identifiant la culture chrétienne qui s'impose de plus en plus, la ruine des cultures précédentes est là pour figurer la dissension et les conflits qui aboutiront à la destruction de la culture présente. C'est dans cette même logique que des éléments ruinés réapparaissent à la fin du film, lorsque, au fil des rues, nous sommes menés jusqu'au pied du phare, charnier des massacres inter-communautaires. La pérégrination urbaine nous conduit métaphoriquement de la ruine architecturale à la décomposition du corps humain, et, partant, à la ruine du corps social. On observe donc dans Agora un traitement particulier de la ruine qui met en place une conjonction entre sa dimension métaphorique et son statut diégétique : elle endosse un rôle proleptique précisément parce qu'elle symbolise les destructions à venir. Cependant, ces ruines, même si leur

24 En ce qui concerne cette représentation du christianisme comme religion fanatique, nous pouvons lire ces remarques : "Délivrant une charge sans concession contre le fanatisme religieux, cette superproduction espagnole n'aurait pas pu être réalisée aux États-Unis : l'histoire fait apparaître le christianisme comme une secte parmi d'autres, qui prend le pouvoir en s'infiltrant dans les failles d'une société décadente dont les élus s'adonnent au divertissement tandis que la population crève la faim », E. LEROY, Film actu.com, URL = [http://cinema.jeuxactu.com/critique-cinema-agora-1-8505.htm], date de consultation le 27 septembre 2012. On lit encore, à propos des acteurs qui interprètent les chrétiens : "Alejandro Amenábar n'est pas un cinéaste très subtil. Il désigne clairement les chrétiens à la vindicte du spectateur. Avec leur grande robe noire, leur barbe et leurs yeux de braise, ces chrétiens-là ressemblent à s'y méprendre à des figures contemporaines. Ce postulat choquera, consolera ou amusera selon la place que l'on occupe dans ce débat millénaire. » Th. Sotinel, "Agora: un péplum intellectuel pour célébrer Hypatie, mathématicienne et païenne ", Le Monde.fr, 05.01.2010, URL = http ://www.lemonde.fr/cinema/ article/2010/01/05/agora-un-peplum-intellectuel-pour-celebrer-hypatie-mathematicienne-et-paienne_1287683_3476.html, date de consultation le 27 septembre 2012. 
statut est intéressant au vu de notre analyse, restent des occurrences minoritaires dans le paysage diégétique d'Agora. Le film d'A. Amenábar figure, dans ce sens, un aboutissement du péplum contemporain dans son utilisation des technologies de l'image virtuelle, en nous proposant une reconstitution totale du paysage urbain alexandrin. C'est à celle-ci que nous allons consacrer notre prochaine partie.

Au terme de cette analyse, il est loisible de constater que la ruine n'occupe plus ni la même place ni la même fonction dans les péplums des années 2010 : un basculement s'opère entre les films du début des années 2000, encore sous influence de l'héritage du péplum classique, qui instrumentalisent la ruine à des fins de marquage temporel ou exotique, et les plus récents de notre corpus, qui réinvestissent la ruine à des fins symboliques. Le développement de l'utilisation cinématographique des nouvelles technologies de l'image dans les fictions antiquisantes libère donc la figure de la ruine de son rôle temporalisant, puisque désormais les seules limites de la reconstitution des paysages et des villes antiques sont la connaissance du passé et l'imagination des décorateurs. C'est la reconstitution totale de villes antiques qui assume le marquage antiquisant du récit, autrefois confié aux ruines, en offrant une représentation spectaculaire de l'Antiquité. De plus, grâce aux mêmes techniques virtuelles, l'Antiquité n'est pas seulement ressuscitée, mais un nouveau regard sur celle-ci trouve une voie d'expression. L'utilisation de caméras virtuelles circulant dans les espaces diégétiques marque une double nouveauté : l'inscription dans l'espace de nouveaux positionnements du regard, et l'inscription dans le temps d'un regard sur l'Antiquité qui l'actualise à travers de nouveaux médiums.

\section{Agora ou les «possibilités du Nil »: de la ville totale à la circulation du regard}

Nous quittons à présent les ruines pour apprécier ce changement de paradigme du péplum contemporain en envisageant, à partir du film Agora, le cas d'une reconstruction totale du paysage urbain qui s'inscrit dans un souci de vérité historique et archéologique. Agora prend pour cadre la plus grande ville de l'Antiquité hellénistique et tardive, la cité égyptienne d'Alexandrie à la fin du IV e siècle après J.-C. La ville est alors déchirée entre l'héritage d'une culture païenne hellénistique et savante et l'émergence d'une pensée nouvelle portée par les religions monothéistes. C'est dans les remous de ces conflits que le réalisateur situe l'action de son film, une biographie-fiction ${ }^{25}$ centrée

25 "Dans une adaptation crédible, le film raconte la vie et la mort de la dernière femme savante de l'Antiquité, même si son réalisateur, privilège de l'artiste oblige, a pris quelques libertés avec la réalité : Synésios survit en effet à Hypatie ; l'assassinat de celle-ci est moins brutal grâce à l'intervention de son ancien esclave qui adoucit les circonstances de sa mort. Mais le plus contestable est le fait de montrer la savante en philosophe quasiment athée, ce qui parait peu vraisemblable [...] », O. Gaudefroy, Hypatie, L'étoile d'Alexandrie, Paris, Arléa, 2012, p. 130-131. 
sur le personnage d'Hypatie d'Alexandrie. Si la ville d'Alexandrie éveille un imaginaire collectif fort, c'est la représentation du paysage urbain en son ensemble qui excède la simple fonction métonymique dont relèvent ses deux monuments les plus emblématiques (le Phare et la Bibliothèque) depuis l'Antiquitée ${ }^{26}$. Beaucoup plus qu'un simple écrin à l'action, Alexandrie, telle que la figure A. Amenábar, se voit, au fur et à mesure de son apparition, prise dans un mouvement plus ample qui, peu à peu, fait de la ville un paysage-monde ${ }^{27}$. Sur ce dernier s'exerce le regard énigmatique d'une instance suprahumaine, par le biais de la construction d'une vision panoptique du paysage. La narration d'Agora se fragmente en une multiplicité de lieux dont la variété montre l'ampleur du travail de reconstitution virtuelle de la ville d'Alexandrie. La diégèse articule ainsi lieux du savoir (Bibliothèque ${ }^{28}$, baie d'Alexandrie), du pouvoir (Sénat), du culte (Separeum et église chrétienne), de l'espace social (agora et amphithêâtre), de l'intimité (maison d'Hypatie et de Théon), de l'issue dramatique de la fiction (contreforts du Phare, sur l'île de Pharos). À l'évidence, l'articulation diégétique de ces différents sites nécessite la mise en place d'une topologie qui les structure, c'est-à-dire qui rend lisible leur relation spatiale. A. Amenábar organise l'espace scénique de l'Alexandrie d'Agora en ayant recours à une double circulation : celle des voies physiques de la circulation urbaine (rues, remparts, portes, etc.), et celle du regard qui embrasse ces différents espaces. Les espaces de circulation sont investis tout à la fois d'une charge dramatique et d'une valeur symbolique et morale : lieux du passage (du légal à l'illégal ${ }^{29}$, de la vie

26 Dès l'Antiquité, cette identification métonymique de la ville d'Alexandrie à son phare ou à sa bibliothèque est présente : en témoigne par exemple la représentation de ces monuments sur des pièces de monnaie romaines frappées par Antonin le Pieux ( $\mathrm{v}^{\mathrm{e}}$ et VIII ${ }^{\mathrm{e}}$ année tribunicienne). Voir à ce sujet H. FraGaKi, Images antiques d'Alexandrie, Le Caire, Institut Français d'Archéologie Orientale, 2011, p. 123.

27 Nous nous contentons, pour l'instant, d'introduire l'expression " paysage-monde ", que nous justifierons par le suite. Toutefois, dès à présent, nous pouvons la rapprocher de la perspective développée par Christian Jacob et François de Polignac, qui consiste à faire de la ville d'Alexandrie un "microcosme du monde ", une "ville œecuménique " (in Alexandrie, III siècle avant J.-C. Tous les savoirs du monde, ou le rêve d'universalité des Ptolémées, Paris, Autrement, 1992, p. 35-81).

28 Notons qu'il s'agit ici de la Bibliothèque Annexe, la première ayant été détruite par un incendie bien avant le temps de la diégèse du film. Dans notre article, nous adopterons pour des raisons de clarté la terminologie du film, en désignant toujours par "Bibliothèque ", la seconde Bibliothèque, c'est-à-dire la Bibliothèque Annexe.

29 C'est par exemple devant la porte du Serapeum qu'est prise, par le collège du Serapeum constitué des prêtres, des savants et du directeur de la Bibliothèque, la décision de répliquer aux affronts des Chrétiens, faisant ainsi basculer les sages dans la résistance, ainsi que dans l'illégalité. De la même manière, c'est devant la porte du rempart du Serapeum qu'est rendue publique la décision de l'Empereur d'amnistier les insurgés mais aussi d'autoriser les Chrétiens à pénétrer dans l'enceinte sacrée. 
à la mort ${ }^{30}$, etc.), ils sont aussi les lieux d'un empressement synonyme de décisions irréfléchies ou arbitraires. Le paysage urbain, dont on voit au début du film des espaces plus vastes, où la parole et les idées circulent encore (agora), se resserre sur ces éléments structurants (ruelles, passages, portes) qui limitent tant la circulation des individus que celle des idées. En restreignant la possibilité de plans larges, A. Amenábar guide le regard vers l'issue inéluctable du film.

Tout à l'opposé de ce resserrement spatial et diégétique, d'autres images nous font accéder à une vision cosmique ou panoptique, qui construit réellement l'image de cette ville-monde. Avec Agora, nous sommes dans le cadre d'une fiction antiquisante qui construit virtuellement une ville totale. Toutefois, il apparait, dès les premières séquences du film, que la perception de cette ville totale ne puisse se faire à l'échelle humaine. Les lieux traversés, l'enchevêtrement des ruelles, montrent autant de perspectives tronquées, d'horizons obturés. Comme s'il fallait s'extraire de la densité grouillante du tissu urbain pour être à même de percevoir le travail de recréation totale du paysage alexandrin. Ce type de regard, qui offre au spectateur la découverte de cette ville totale, existe dans Agora de différentes manières. Deux niveaux d'élévation de la caméra au-dessus du tissu urbain nous permettent de découvrir la totalité de la ville. Premièrement, le niveau médian des remparts, des toits et du phare, soit des structures urbaines qui permettent au regard humain de s'élever. Deuxièmement, une vision en plongée totale sur la ville, qui ne peut logiquement résulter d'une vision humaine.

\section{Remparts, toits et phare}

Tout comme les autres espaces de circulation urbaine que nous avons mentionnés plus haut (rues et portes), les remparts ${ }^{31}$ participent de la structuration topologique de l'Alexandrie d'A. Amenábar. Leur caractère hybride (clôture mais aussi voie de circulation) est instrumentalisé de manière singulière. On y retrouve un topos de la littérature épique : la vision teichoscopique ${ }^{32}$, soit celle des assiégés lorsqu'ils montent

30 Lors de la marche forcée d'Hypatie sous escorte militaire, celle-ci est conduite au Serapeum devenu église. Une séquence focalise sur la montée des marches et le franchissement de la porte, dernière porte ouverte par Hypatie avant qu'elle ne se referme sur sa vie.

31 Ils sont une importante partie de l'infrastructure urbaine pensée par les premiers Ptolémées, voir à ce propos Y. Garland, "Les murailles d'Alexandrie ", in Collectif, $\mathrm{La}$ Gloire d'Alexandrie, Paris, Édition des Musées de la ville de Paris, 1998, p. 87-88.

32 Comme le note Christian Jacob, "Dès l'Iliade et l'Odyssée, nous relevons l'importance de la skopiè, sommet de montagne mais aussi " guette ", " observatoire ", lieu d'où les dieux regardent les combats des hommes, d'où l'homme surveille l'approche de vaisseaux ou d'un banc de poissons. Le lexicographe Hésychius, s.v. Skopiè, glose : «lieu élevé d'où il est possible de voir et de regarder autour de soi ». Le regard aérien, ici, n'a rien de magique : il permet de découvrir un panorama relativement limité (un champ de bataille, une région, un bras de mer). Pour jouir d'une vue d'ensemble sur la terre, il faudrait entasser 
sur les remparts afin d'observer leurs ennemis ${ }^{33}$. C'est le cas, par exemple, lorsque les savants de la Bibliothèque, reclus dans l'enceinte du Serapeum, observent les chrétiens qui les assaillent ${ }^{34}$. Mais les remparts sont aussi le lieu de la contemplation du cosmos, qui peut mener à l'élaboration théorique (n'oublions pas ce sens possible du mot theoria, contemplation). Ainsi, nous entendons la conversation nocturne entre Hypatie et Hésychios juché sur les remparts, qui porte précisément sur la nature des corps astraux, et interroge l'opposition entre la conception héliocentriste d'Aristarque et celle de Ptolémée. De la même manière, les toits rendent plus proches les cieux, auxquels s'adresse l'esclave Davius lors d'une scène de prière, depuis la terrasse du toit de la maison d'Hypatie. Toit sur lequel on retrouvera Hypatie, quelques heures avant son exécution, remerciant le ciel de lui avoir permis de confirmer la conception d'Aristarque, en découvrant que la terre décrit non pas un cercle mais une ellipse autour du soleil. C'est d'ailleurs à cette démonstration que s'emploie Hypatie dans une séquence qui nous fait découvrir les contours de la cité vus depuis le large. La scène commence par une vision de très haut de la baie, prise à la même altitude que le phare. La caméra virtuelle tourne autour du phare, dont on peut apprécier la reconstitution 3D, puis repère au large l'embarcation d'Oreste et d'Hypatie et nous dirige vers elle. Le plan du phare, peu pertinent d'un point de vue diégétique, l'est bien plus en revanche du

plusieurs montagnes les unes sur les autres : alors l'altitude serait suffisante ", in "Dédale géographe. Regard et voyage aériens en Grèce ", Lalies 3 (1981), p. 150.

33 La circulation du regard est également construite par des jeux de caméra depuis une hauteur, voire depuis le cosmos lui-même. Les paysages panoptiques et les visions cosmiques d'Agora que nous venons de décrire peuvent être rapprochés d'une modalité de regard antique qui apparaît durant l'époque hellénistique et tardive : un type de regard précisément paysager qui émerge au croisement de la littérature et de la géographie hellénistiques. En effet, on observe, dans cette utilisation singulière des mouvements vertigineux de caméras, réelles ou virtuelles, quelque chose de comparable à ce que $\mathrm{D}$. Meyer nomme bird's eyed view ("This geography reflects a wish for a bird's eyed view of a known stretch of distant land, as if one were looking down at it on a map. Especially the relative position of various locations makes sense only in terms of such a quasi-cartographic overview, an experience one can have in several places in mountainous Greece. Various forms of such flight and hiking fantaisies are to be found in Greek literature. They make possible the visualization of an imaginary map and thereby not only the orientation of the reader but also the appreciation of the landscape's extent. »: D. MEYER, "Apollonius as a Hellenistic geographer ", in Th. D. Papanghelis et A. Rengakos (éd.), A Companion to Apollonius Rhodius, Leiden, Boston, Köln, Brill, 2011, p. 231), ou à ce que Ch. Jacob, quant à lui, appelle "regard de Dédale " (voir à ce propos Ch. JАСОВ, "Dédale géographe. Regard et voyage aériens en Grèce ", op. cit., p. 154, ou encore L'empire des cartes. Approche théorique de la cartographie à travers l'histoire, Paris, Albin Michel, 1992, p. 210). L'invention d'un regard abstrait, au moment où naissent les premières représentations cartographiques et paysagères du monde, va être instrumentalisée par la littérature et par la géographie, afin de construire une image cohérente du monde en s'élevant de sa surface.

34 Plus tard, c'est toujours depuis les remparts de l'enceinte du Serapeum que les savants assistent à la lecture de l'acte de délivrance prononcé en leur faveur par l'Empereur. 
point de vue métonymique : comment, en effet, imaginer un film sur Alexandrie sans son célèbre phare!

Qu'il s'agisse des toits, des remparts ou du bateau, on note une proximité dans la position des personnages qui s'y tiennent. Ceux-ci s'élèvent (ou s'éloignent) au-dessus (au-delà) de la masse urbaine et de ses foules hostiles, dans une posture statique qui contraste avec l'agitation de la ville et les flux oppressants des voies de communication. Ces espaces surplombants apparaissent dès lors comme des lieux désengagés de la lutte des passions, des lieux de calme et de pensée, situés à mi-chemin entre le monde des hommes et le monde supra-céleste que nous allons maintenant envisager.

\section{Visions panoptique et cosmique}

La scène d'ouverture d'Agora, avant l'apparition du titre, laisse apparaître un fond de nuit étoilée ${ }^{35}$, sur lequel se surimprime le texte présentant le contexte historique du film. La caméra virtuelle poursuit un mouvement rotatif qui, semble-t-il, s'apparente à la révolution des corps astraux, pendant que l'on entend la voix d'Hypatie qui professe sa leçon d'astronomie, soulignant le mouvement de la caméra en affirmant que le cercle est la figure géométrique la plus parfaite qui soit ${ }^{36}$. À la faveur de ce mouvement circu-

35 Il y a ici ambiguïté dans la position supposée du regard caméra : nous pourrions aussi bien être sur terre que dans le cosmos. Toutefois, le mouvement de caméra lève rapidement cette ambiguïté : nous sommes dans l'espace.

36 L'ouverture depuis les cieux semble être appréciée des metteurs en scène pour situer le contexte de leurs films antiquisants. On pense ici notamment à une exploitation littérale de la bird's eye view (cf. note 33), largement répandue dans les films de notre corpus, à la faveur de laquelle la caméra suit un oiseau pour dynamiser cette vision céleste et/ou cosmique. Tout d'abord, dans Le Choc des Titans, de Louis Leterrier, l'histoire s'ouvre sur fond d'images cosmiques, nébuleuses colorées dont les nappes de gaz se recomposent petit à petit pour former l'image des constellations à l'effigie des dieux, des déesses et des héros. On comprend, à la faveur du raccord avec le plan suivant, que ces images sont en fait un reflet, pris dans l'œil de l'une des innombrables statuettes ornant l'Olympe. Ces statuettes s'avèrent être des effigies de chacun des humains qui vivent sur la terre : ainsi, lorsque l'on pensait pouvoir interpréter les images cosmiques comme celles de la toutepuissance des dieux contemplant le monde des hommes depuis leur séjour éthéré, ce sont finalement les hommes qui, par le truchement des effigies, observent le monde de leurs créateurs. Retournement intéressant que celui-ci, opéré par l'instrumentalisation des jeux de regard et l'habileté du travail de montage. Ensuite, toujours dans Le Choc des Titans, c'est un aigle, avatar de Zeus, que nous retrouvons pour construire la circulation du regard dans l'espace. L'oiseau nous conduit depuis l'espace aérien de l'Olympe que nous avons évoqué plus haut, nous faisant découvrir au passage toute sa richesse architecturale, jusqu'à - à travers la mer de nuages - la terre où se déroule le drame humain. Le volatile, que nous retrouvons à certains moments $\mathrm{du}$ film, y incarne à chaque fois la présence de Zeus qui observe son protégé Persée. Enfin, un même procédé se retrouve dans Les Immortels, de T. Singh (2011), à travers l'utilisation de deux types d'oiseaux qui sont mis 
laire, la planète Terre entre dans le champ, faisant apparaître le delta du Nil, le continent africain et la péninsule arabique. Puis ce mouvement rotatif poursuit sa course dans le système solaire, laissant entrevoir la lune. Un habile jeu sur la forme sphérique (qui va se répéter tout au long du film) fait apparaître un cercle dont les contours sont flous : il pourrait s'agir du reflet de la lune sur une étendue d'eau. Cette forme vient constituer le $\mathrm{O}$ du titre Agora qui s'y superpose, nous faisant ainsi passer du système solaire à la terre. La focalisation de la caméra sur la forme circulaire blanche substitue ensuite le soleil à la lune, et par un mouvement de dézoom, nous fait pénétrer à reculons, dans la salle où Hypatie délivre son enseignement, désacousmatisant dès lors sa voix. C'est ici la première image que nous présente $\mathrm{A}$. Amenábar de l'intérieur de la Bibliothèque (une salle de cours). À ce plan inaugural de l'espace diégétique du film, succède un large plan d'ensemble qui instaure les divers bâtiments de l'enceinte en un site unifié, identifié comme Bibliothèque par un titre qui légende le site. Ce plan n'est pas filmé à hauteur d'homme; il ne peut pas non plus, en toute logique, être vu depuis une hauteur accessible à l'homme (tel un rempart ou un toit en terrasse). Ce plan semble être vu par la même "instance " que la séquence cosmique précédente. Après qu'un mouvement ascensionnel de la caméra nous a révélé l'ensemble du site, nous y pénétrons cette fois à hauteur d'homme. Quelques plans rapprochés montrent la porte de l'enceinte, une scène de prière devant le Serapeum, et font de la Bibliothèque un lieu vivant de culte et de savoir. Enfin, nous pénétrons l'espace proprement dit de ce que nous, modernes, entendons par bibliothèque ${ }^{37}$ : à savoir, le lieu de conservation des ouvrages, lequel

en concurrence : les faucons d'Hypérion, qui cherche à libérer les Titans afin de mettre fin à l'empire des dieux sur le monde, et les aigles, traditionnellement associés à Zeus, qui représente la lutte pour maintenir l'ordre du monde tel qu'il est, et en paix.

37 Si la représentation que donne A. Amenábar de la Bibliothèque d'Alexandrie s'écarte parfois de la réalité historique (notamment au niveau des intérieurs et du portique de l'enceinte), la reconstruction reste appréciable en ce qu'elle présente des caractéristiques véridiques que les plans de caméra exploitent habilement. Ainsi, le système de classement des papyri mis en scène dans le film est bien celui de la réalité historique : classés par thèmes, les ouvrages étaient étiquetés avec le nom de l'auteur et le titre de l'œuvre, facilitant l'exploitation d'un tel fond. Toutefois, si nous avons connaissance du système de classement des ouvrages, nous ne disposons que de peu d'informations concernant la décoration de ce lieu. Ainsi, la présence de bustes de facture plutôt romaine que grecque, de la mosaïque du parterre à l'effigie d'Alexandre, et des fresques de la coupole représentant les signes du zodiaque est un parti pris de décorateur, certes destiné à faire couleur locale, mais relativement crédible. Nous émettons cependant des réserves au sujet du placement dans le décor diégétique de la mosaïque à l'effigie d'Alexandre. Sans nous lancer dans la fiction archéologique, précisons tout de même que ce portrait d'Alexandre, représentant le souverain avec les cornes de l'oracle d'Amon, est la reproduction de celui que représentent nombre de monnaies, et en ce sens une image largement répandue dans l'Égypte de cette période. Néanmoins, son placement dans la Bibliothèque annexe semble peu probable dans la mesure où il n'a mis en œuvre ni le chantier de la première Bibliothèque, ni, a fortiori, le chantier de la seconde Bibliothèque. 
nous est révélé par un ample mouvement circulaire de caméra, qui redouble la circularité formelle de l'espace de la bibliothèque, et poursuit ce jeu sur la forme sphérique dont nous avons souligné l'emploi plus haut. Cette séquence d'ouverture inaugure le dialogue entre visions cosmiques du monde et visions panoptiques d'Alexandrie. En articulant ces deux types de regard, A. Amenábar institue la Bibliothèque, centre de la ville-monde qu'est Alexandrie, en puissance ordonnatrice du récit.

D'autres séquences du film poursuivent ce travail et attestent la force centripète de la Bibliothèque. Lors de la scène du saccage de la Bibliothèque par les Chrétiens, ce jeu formel se prolonge. Il se produit alors une association entre l'espace sphérique de la Bibliothèque, le cercle formé par de la mosaïque d'Alexandre au sol, la forme de l'oculus, et la maquette du système solaire fabriquée par Davius, l'esclave-assistant d'Hypatie. Dès lors que Davius brise la maquette en la fracassant contre une fenêtre, c'est le rejet définitif de la philosophie et de la science païennes qui est signé : l'ordre du monde s'inverse alors. La caméra monte vers l'oculus encadré de représentations zodiacales (référence supplémentaire au système stellaire et à l'ordonnancement du monde en un cosmos qui s'oppose au chaos qui est en train d'advenir) dans un mouvement de contre-plongée totale, pendant que des volumina volent dans les airs au-dessus de l'objectif. Toujours dans le même plan, le mouvement de caméra s'inverse et nous finissons de contempler le saccage la tête en bas, en image inversée. Le plan suivant renoue avec nos images panoptiques, en nous permettant de découvrir l'extérieur de la Bibliothèque, vu de très haut, cette fois en plongée totale, centrée à nouveau sur l'oculus dans un effet de champ-contrechamp. Le plan d'ensemble laisse apparaître l'enceinte de la Bibliothèque, dans laquelle se répandent les hordes de chrétiens qui jettent des volumes au feu. Plus tard dans le film, nous retrouvons, de loin, le feu continu des autodafés. Cette autre séquence débute au ras du sol, dans une rue, devant un héraut qui proclame la condamnation définitive des cultes païens. La caméra décrit ensuite un mouvement ascensionnel qui s'extrait d'abord lentement de l'espace de la rue et s'achève en laissant entrevoir au loin la fumée montant de la Bibliothèque. Après un fondu au noir, apparaît un plan cosmique qui laisse voir la terre depuis le ciel. On y repère tout de suite le delta du Nil, tandis qu'un texte y apparaît pour indiquer une ellipse temporelle. Un mouvement continu de zoom avant, semblable au type d'images que permet le logiciel Google Earth, s'avance depuis le cosmos vers Alexandrie, en nous laissant deviner son plan d'urbanisme. Le zoom se prolonge jusqu'à laisser apparaître la Bibliothèque désormais pacifiée, d'abord l'enceinte et tous les bâtiments qu'elle recèle, jusqu'à nous laisser pénétrer dans le Serapeum devenu église dans l'intervalle.

Nous voici de nouveau confrontés à l'articulation entre ces deux regards, panoptique et cosmique. Les possibilités techniques offertes par l'image virtuelle permettent ainsi une représentation totale de la ville d'Alexandrie, laquelle n'est pas seulement reconstituée physiquement dans sa forme, mais aussi dans la vraisemblance de sa géographie et de son plan urbanistique, ainsi que dans son évolution temporelle, puisque ces déplacements nous donnent accès à ses différentes époques. Toutefois, ce regard en plongée totale n'est pas seulement un regard qui construit la matérialité 
spatiale et temporelle d'Alexandrie, encore assume-t-il des enjeux symboliques qu'il faut à présent comprendre.

Ce regard, tel qu'il est mis en œuvre par Amenábar dans Agora, pose la question de l'identité de celui qui le porte. $\mathrm{Si}$, dans les autres films de notre corpus, où nous avons pu repérer une utilisation similaire de ce type de vue, ce regard pouvait se comprendre comme celui des dieux contemplant le drame humain (nous allons le voir plus bas), dans Agora, le réalisateur ménage une place au doute. On pourrait comprendre ce regard comme celui des différents dieux des diverses religions qui s'affrontent dans le film (dieux païens, dieu juif ou chrétien). Néanmoins, nous proposons une interprétation différente - ou complémentaire. Il nous semble en effet que ce regard fortement distancié dans l'espace le soit aussi dans le temps : les révolutions astrales " parcourues » par la caméra virtuelle désigneraient tout autant de l'espace que du temps. Ainsi, le regard cosmique porté sur l'Alexandrie ressuscitée peut être le nôtre, celui du spectateur contemporain, qui assiste depuis son époque à la renaissance de l'Antiquité.

Dans tous ces films des années 2000-2010, qu'ils s'apparentent au péplum, à la biographie-fiction historique ou bien qu'ils relèvent de la fantasy antique, on repère cette constance du recours au regard zénithal. Celui-ci matérialise, pour les réalisateurs, une possibilité de structuration de l'espace diégétique par des jeux de caméras virtuelles. L'irruption de ce regard abstrait, omnipotent, qui amplifie celui du spectateur, est le moyen cinématographique de nouvelles possibilités de raccords entre les différents plans, et apporte une cohérence aux relations topologiques des divers lieux représentés. Il est aussi l'occasion de la naissance de nouveaux paysages de l'Antique, dont les enjeux représentationnels circulent entre un regard proprement antique, au cœur de la vision du monde des Anciens, et les actualisations contemporaines de ce regard (on pense ici à l'imagerie offerte par certains logiciels contemporains, Google Earth en tête). On remarque en outre une circulation de ces jeux de regard d'un film à un autre, dans une sorte d'intertextualité filmique qui se développe autour de la résurrection cinématographique des villes et des paysages antiques.

Quelle que soit la motivation réelle de ces choix, sur laquelle nous ne pouvons malheureusement que spéculer, leur résultat, pour le spécialiste, est bien un effet de " couleur locale " qui est plutôt de l'ordre du kairos ou de l'hermaion. Cette façon de construire l'espace pour ensuite illustrer le monde par des paysages précis, relève d'une attitude face au monde qui s'est structurée à la période hellénistique : la cartographie a fait naitre un type de regard porté sur le monde, et le mode de représentation du paysage par les Anciens qui en découle nourrit encore nos modélisations contemporaines.

Malgré les critiques qui cantonnent ces films à la catégorie du divertissement de large audience - certes, ce ne sont pas tous de bons films, mais notre propos n'est pas de juger de leur qualité artistique - ils sont pour nous l'indice de la justesse du propos de Marc Fumaroli, lorsqu'il écrit qu' 
"Antique signifie en réalité non d'autrefois, mais de toujours. C'est la lumière qui ne change pas. C'est le temps vainqueur du temps, dont nous sommes devenus pauvres et dont aujourd'hui nous avons besoin ${ }^{38}$."

S’appropriant les technologies les plus innovantes, le cinéma est aujourd'hui le lieu d'un changement de paradigme qui, à l'heure de la " mort programmée » de l'Antiquité, n'identifie plus celle-ci à un champ de ruines mais, au contraire, à un espace à reconstruire ou à réinvestir, à un paysage à explorer et à re-composer. Dans l'imaginaire des réalisateurs, si la part du mythe, et la part réelle, archéologique, de l'Antiquité, sont confondues, c'est parce qu'elles relèvent en fin de compte du même matériau plastique. Fictionnés, métissés, hybridés, le mythe, l'Histoire et le matériel archéologique forment la matière que les fictions antiquisantes contemporaines modèlent et remodèlent, dans le travail de refonte et de recréation de l'Antiquité d'aujourd'hui, qui lui accorde, toujours, la place fantasmatique de nos origines.

Laury-Nuria ANDRÉ

Docteur en Langues et Littératures anciennes, chargée de cours à l'ICT

80 allées Jean Jaurès App. 1104

F-31000 Toulouse

laury.andre@ens-lyon.fr

Sophie Lécole SolnychKInE

MCF en Arts Plastiques,

Université de Toulouse 2 (UTM)

6 allée Paul Sabatier

F-31000 Toulouse

sophiesolnychkine@free.fr

38 M. Fumaroli, L'État culturel, essai sur une religion moderne, Paris, Éditions de Fallois, 1991. 